\title{
BICYCLIST BLACK CARBON EXPOSURE AND THE IMPACT OF ROUTES CHOICE AND BIKING FACILITIES, A SPATIAL ANALYSIS
}

Luc Dekoninck, Ghent University, department of Information Technology, St-Pietersnieuwstraat 41, 9000 Ghent, Belgium

Prof. dr. Ir Dick Botteldooren, Ghent University, department of Information Technology, StPietersnieuwstraat 41, 9000 Ghent, Belgium

Luc Int Panis, Flemish Institute for Technological Research (VITO), Boeretang 200, Mol, Belgium and Transportation Research Institute Hasselt University, Wetenschapspark 5 bus 6, Diepenbeek, Belgium

Background and Aims: Personal exposure to Black Carbon is strongly related to activity pattern and more specifically linked to the time spent in traffic related micro-environments. Enhancing bicycle use is a popular policy measure but has a risk of increasing exposure to air pollution.

Methods: A mobile measurement campaign was set up in the city of Ghent, Flanders to explore the spatial and temporal variability of Black Carbon (BC) exposure for bicyclists in urban and suburban areas. More than 100 commuting trips ( $>=8 \mathrm{~km}$ ) were cycled while carrying a microaethalometer over a time span of several months.

Results: The focus of the study is on the spatial and temporal variability of BC exposure and we investigate how the variation relates to geographic, meteorological and local traffic volume parameters. This analysis allowed showing that the exposure to BC can be significantly different when specific cycling facilities are used. Although, during the lowest exposure parts of the individual trips, a significant contribution from background concentrations as observed by the national air pollution monitoring network could be shown, our results clearly indicate that local sources are rather important and thus a cyclist can affect its exposure by careful route choice. Of particular interest are BC concentrations in the vicinity of highways where an influence of wind direction and screening by buildings and noise barriers was observed. Increased BC concentrations are also observed while passing through street canyons with high traffic loads, as expected.

Conclusions:The spatial and temporal evaluations are used to identify the potential relevant geographic parameters to build a general exposure model for bicyclists. In most cases stronger variations were found for the black carbon than was expected from known spatial variations of UFP en PM2.5.A geo-statistical analysis is performed to support these preliminary findings. 